Article

\title{
Effects of Danshensu and Salvianolic Acid B from Salvia miltiorrhiza Bunge (Lamiaceae) on Cell Proliferation and Collagen and Melanin Production
}

\author{
Yi-Shyan Chen ${ }^{1}$, Shu-Mei Lee ${ }^{2}$, Ying-Ju Lin ${ }^{3,4}$, Shu-Hua Chiang ${ }^{5, *}$ and Chih-Chien Lin ${ }^{1, *}$ \\ 1 Department of Cosmetic Science, Providence University, 200 Chung-Chi Road, Shalu, \\ Taichung 43301, Taiwan; E-Mail: yishyan@gm.pu.edu.tw \\ 2 Department of Cosmetic Science and Management, Mackay Medicine, Nursing and Management \\ College, 92 Shengjing Road, Beitou, Taipei 11260, Taiwan; E-Mail: s107@eip.mkc.edu.tw \\ 3 Department of Medical Research, China Medical University Hospital, 2 Yuh-Der Road, \\ Taichung 40447, Taiwan; E-Mail: yjlin.kath@gmail.com \\ 4 School of Chinese Medicine, China Medical University, 91 Hsueh-Shih Road, Taichung 40402, Taiwan \\ 5 Department of Food and Beverage Management, Taiwan Hospitality and Tourism College, 268 \\ Chong-Hsing St., Feng-Shan Village, Shou-Feng County, Hualien 974, Taiwan
}

* Authors to whom correspondence should be addressed; E-Mails: shchiang@mail.tht.edu.tw (S.-H.C.); chchlin@pu.edu.tw (C.-C.L.); Tel.: +886-3-865-3906 (ext. 862) (S.-H.C.);

Fax: +886-3-865-3000 (S.-H.C.); Tel.: +886-4-2632-8001 (ext. 15409) (C.-C.L.);

Fax: +886-4-2631-1167 (C.-C.L.).

Received: 4 December 2013; in revised form: 1 February 2014 / Accepted: 8 February 2014 / Published: 13 February 2014

\begin{abstract}
Danshensu (DSU) and salvianolic acid B (SAB) are the primary water-soluble compounds of Salvia miltiorrhiza Bunge (Lamiaceae). In this study, we analyzed the effects of DSU, SAB and a S. miltiorrhiza extract (SME) on cell proliferation. Additionally, the effects of DSU and SAB on collagen synthesis in Detroit 551 human normal fibroblast cells and on melanin production in B16 melanoma cells were verified. The results demonstrated that SME can enhance the proliferation of Detroit 551 cells and that this boost may be caused by DSU and SAB. This research showed that SME, DSU and SAB all have the ability to increase the production of collagen in Detroit 551 cells. The results also confirmed that DSU and SAB can attenuate the $\alpha-\mathrm{MSH}$-stimulated melanin production of B16 cells by inhibiting tyrosinase activity. Therefore, SME, DSU and SAB each have the potential to be utilized as active ingredients in wound healing or cosmetic treatments. In the future, DSU and SAB could also be used as functional components for treating hyperpigmentation.
\end{abstract}


Keywords: collagenesis; danshensu; melanogenesis; salvianolic acid B; Salvia miltiorrhiza

\section{Introduction}

Salvia miltiorrhiza Bunge (Lamiaceae), generally known as danshen, is an important and widely used medicinal plant in Traditional Chinese Medicine (TCM). It is used in many countries, including China, Korea and Japan [1]. According to the principles of TCM, the dried root of S. miltiorrhiza can be used to promote blood flow and to resolve blood stasis [2]. The beneficial effects of S. miltiorrhiza on the cardiovascular system have been broadly demonstrated [3,4]. The chemical components of an extract of $S$. miltiorrhiza are classified into two main groups: water-soluble (hydrophilic) phenolic compounds and lipid-soluble (nonpolar, lipophilic) diterpenoidal compounds. These active components of $S$. miltiorrhiza, including tanshinones, tanshinlactone, salvianolic acids and danshensu, have potent antioxidant, antimicrobial, anticancer and cardioprotective functions [5-7]. Danshensu (DSU), salvianolic acid A (SAA) and salvianolic acid B (SAB) are phenolic caffeic acid derivatives, and they are the primary water-soluble compounds of $S$. miltiorrhiza. Among them, danshensu and salvianolic acid B are present in the highest concentrations [8]. Their structures are shown in Figure 1. Danshensu is a relatively simple phenolic acid found in the S. miltiorrhiza extract that exhibits cardiovascular protective effects. Additionally, danshensu acts on human umbilical vein endothelial cells to protect against injuries and inhibit the production of ROS, effects that have been established by several studies $[7,9,10]$. The therapeutic potential of salvianolic acid B in hepatic protection, neural protection and cancer treatment has been proposed in recent years, although the compound's greatest clinical impact is in cardiovascular protection [11-14]. However, the effects of danshensu and salvianolic acid B on the regulation of collagenesis and melanogenesis have not been fully studied.

Figure 1. The chemical structures of (A) danshensu and (B) salvianolic acid B.

(A)

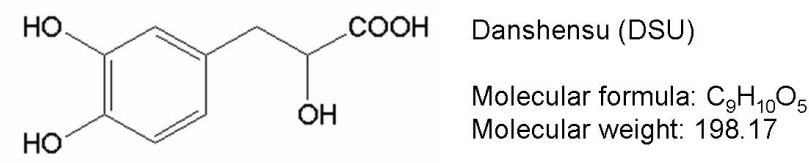

(B)

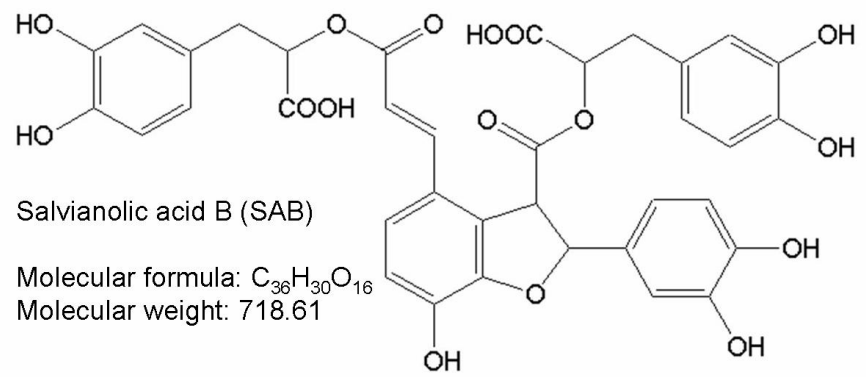

The synthesis of melanin in melanocytes is catalyzed by melanogenic enzymes, including tyrosinase, tyrosinase-related protein 1 (TRP-1) and tyrosinase-related protein 2 (TRP-2). These 
enzymes play critical roles in melanin production via the hydroxylation of tyrosine into dihydroxyphenylalanine (DOPA) and the further oxidation of DOPA into DOPAquinone. The increased activity of these enzymes, caused by stimulative factors such as ultraviolet (UV) light and chronic inflammation, may lead to hyperpigmentation. Therefore, melanogenesis inhibitors can be used to treat hyperpigmentation in skin $[15,16]$.

Collagens are the most abundant proteins in mammals; they play structural roles, contributing to the mechanical properties, organization and shape of tissues. Collagens interact with cells to regulate their proliferation, migration, and differentiation [17]. In the collagen superfamily, type I collagen is the most rich extracellular matrix protein and is essential for the mechanical strength of tissues [18]. Thus, the regulation of collagen production has the potential to treat the tissue disorders that are related to collagens.

In this study, we analyzed the effects of danshensu and salvianolic acid B on collagen synthesis in Detroit 551 normal fibroblast cells and on melanin production in B16 melanoma cells. Additionally, the effects of danshensu, salvianolic acid B and a S. miltiorrhiza extract on cell proliferation were tested.

\section{Results and Discussion}

\subsection{Effects of SME, DSU and SAB on the Proliferation of Fibroblast Cells}

For the preparation of $S$. miltiorrhiza extract (SME), the dehydrated $S$. miltiorrhiza leaves were homogenized and then extracted with water at room temperature for $30 \mathrm{~min}$. The prepared SME should contain plenty of water-soluble (hydrophilic) phenolic compounds, including danshensu (DSU) and salvianolic acid B (SAB). In this study, we first evaluated the effects of DSU, SAB and SME on the growth of Detroit 551 human normal fibroblast cells. The results are shown in Figure 2. As seen in Figure 2A,B, the viability of the Detroit 551 cells in both the low $(0.2 \%)$ and normal (10\%) FBS conditions increased when the cells were treated with different concentrations of SME. The enhanced cell viability of the group treated with $1 \mu \mathrm{g} / \mathrm{mL}$ SME in $0.2 \%$ FBS was approximately $150 \%$ of the control, the highest viability level observed (Figure 2A). Although the cell viabilities of the SME treated group in $10 \%$ FBS were only approximately $120 \%$ of the control, the cell proliferationenhancing activity of SME in that condition was still observed (Figure 2B). When treated with DSU, the increased viabilities of the Detroit 551 cells in both FBS conditions were similar to those of the SME groups. Additionally, the increased levels observed in the DSU groups with either $0.2 \%$ or $10 \%$ FBS were nearly the same (Figure 2C,D). When treated with $100 \mu \mathrm{M}$ DSU, the cell viability increased to $137 \%$ of the control (Figure 2D). Therefore, our results demonstrated that SME and DSU can improve the cell proliferation of Detroit 551 cells in both $0.2 \%$ and $10 \%$ FBS.

The growth of cells treated with SAB was only enhanced in 10\% FBS. Under those conditions, the cell viability levels were approximately $120 \%$ of the control (Figure $2 \mathrm{~F}$ ). However, under $0.2 \%$ FBS conditions, treatment with $100 \mu \mathrm{M} \mathrm{SAB}$ enhanced the growth of the Detroit 551 cells but not significantly (Figure 2E). Our results established that SME has a growth factor (GF)-like ability to enhance the growth of human normal fibroblast cells and that this effect might be caused by the components DSU and SAB. Earlier study has demonstrated that several human cancer cell lines exhibited decreased cell viability when exposed to $125 \mu \mathrm{M} \mathrm{SAB}$ for $24 \mathrm{~h}$ [11]. Moreover, our results 
also revealed that the effects of DSU and SAB on cell proliferation will be attenuated when the concentrations of are higher than $200 \mu \mathrm{M}$ (data not shown). Thus, in this study, we selected $100 \mu \mathrm{M}$ as the highest concentration for our experiments.

Figure 2. The effects of a S. miltiorrhiza extract (SME), danshensu (DSU) and salvianolic acid B (SAB) on the cell proliferation of Detroit 551 cells: (A) SME in 0.2\% FBS; (B) SME in $10 \% \mathrm{FBS}$; (C) DSU in $0.2 \% \mathrm{FBS}$; and (D) DSU in $10 \% \mathrm{FBS}$; (E) SAB in $0.2 \% \mathrm{FBS}$, and $(\mathbf{F}) \mathrm{SAB}$ in $10 \% \mathrm{FBS}$. Each value represents the mean $\pm \mathrm{SE}(n=3)$. $* p<0.05$, compared with the control.

(A)

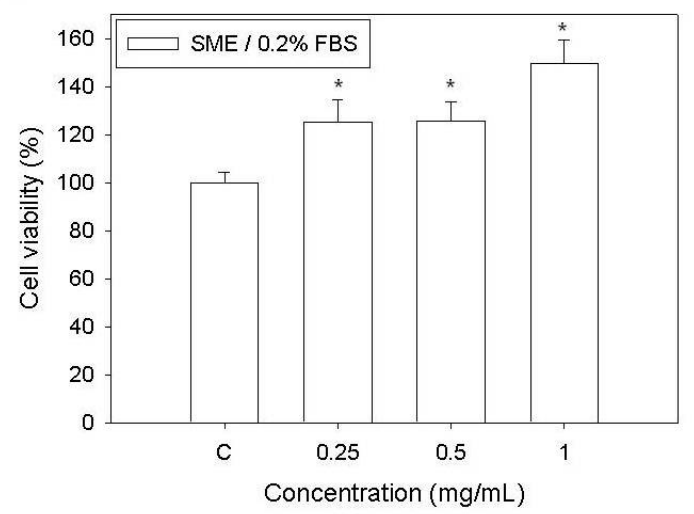

(C)

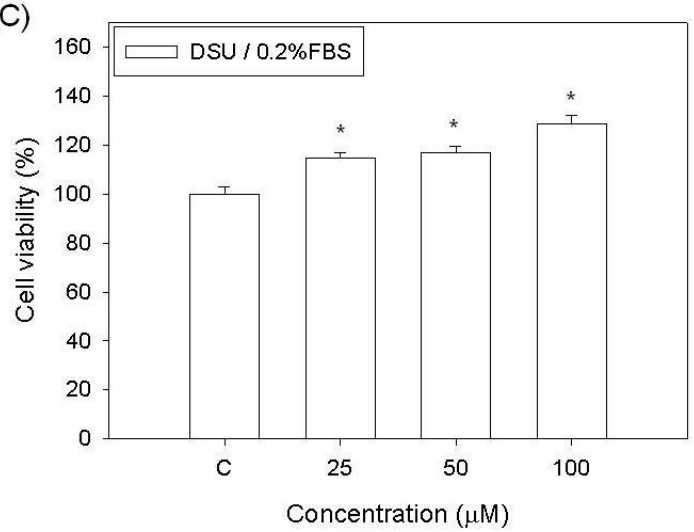

(E)

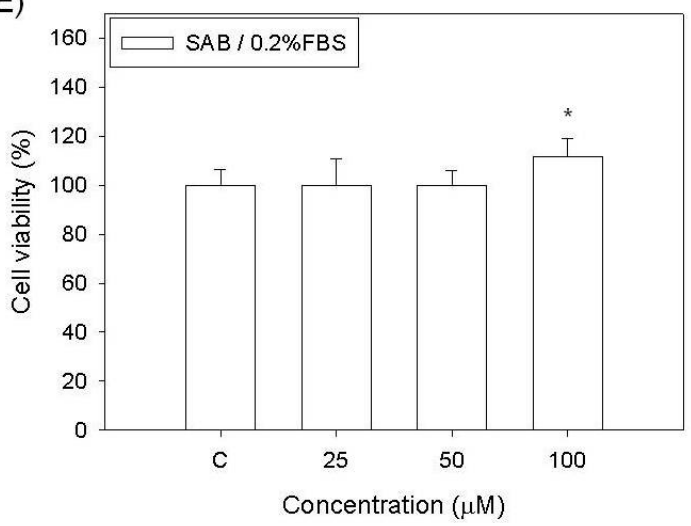

(B)

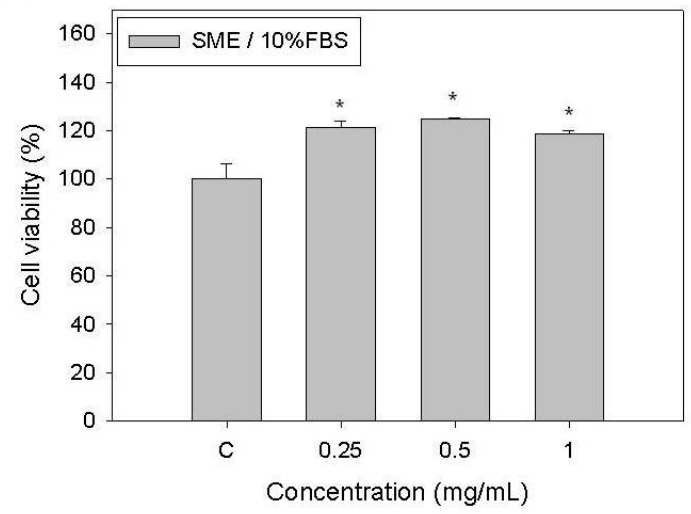

(D)

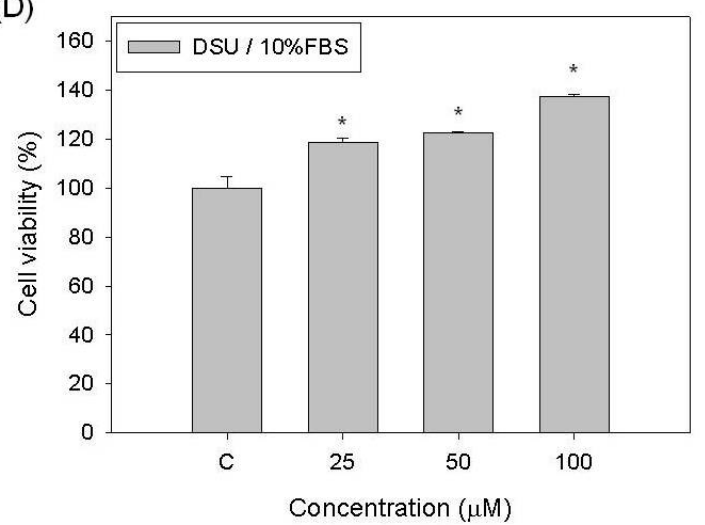

(F)

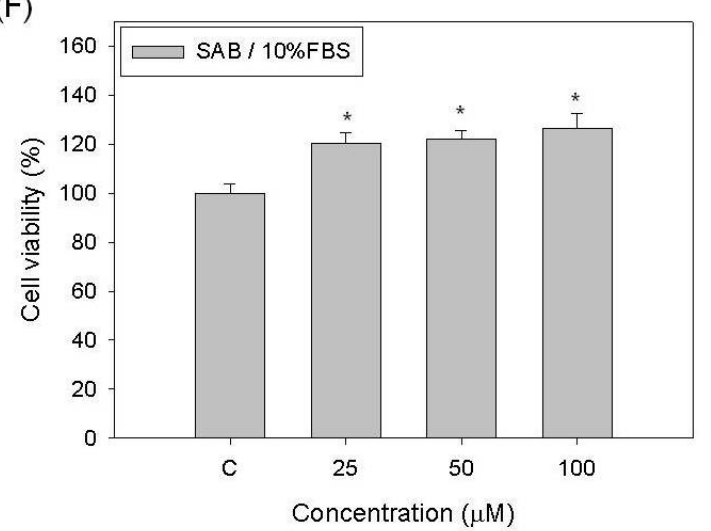

Many functional materials have demonstrated an ability to enhance cell proliferation. For wound healing purposes, a cell growth-enhancing activity may help rehabilitate cut tissues in a shorter time. Additionally, cell activation is a key property for cosmetic skin morphogenesis [19-21]. Therefore, 
SME, DSU and SAB have the potential to be used as functional ingredients in wound healing or cosmetic treatments.

\subsection{Effects of DSU and SAB on the Proliferation of EGF-Treated Fibroblast Cells}

To further confirm the functions of DSU and SAB on the proliferation of fibroblast cells, we tested the cell viability of Detroit 551 cells co-treated with DSU or SAB and human epidermal growth factor (EGF). The results are shown in Figure 3. Our results demonstrated that DSU and SAB cannot enhance the cell proliferation of EGF-treated Detroit 551 cells at any of the concentrations used. The cell viabilities of all EGF-treated groups are approximately $125 \%$ to $130 \%$ of the control (Figure 3 ). Therefore, we can suggest that the effects of DSU and SAB on the proliferation of fibroblast cells are related to the signaling pathway of EGF.

Figure 3. The effects of (A) danshensu (DSU) and (B) salvianolic acid B (SAB) on the cell proliferation of epidermal growth factor (EGF)-treated Detroit 551 cells in $0.2 \%$ FBS. Each value represents the mean $\pm \operatorname{SE}(n=3)$. ${ }^{*} p<0.05$, compared with the control.

(A)

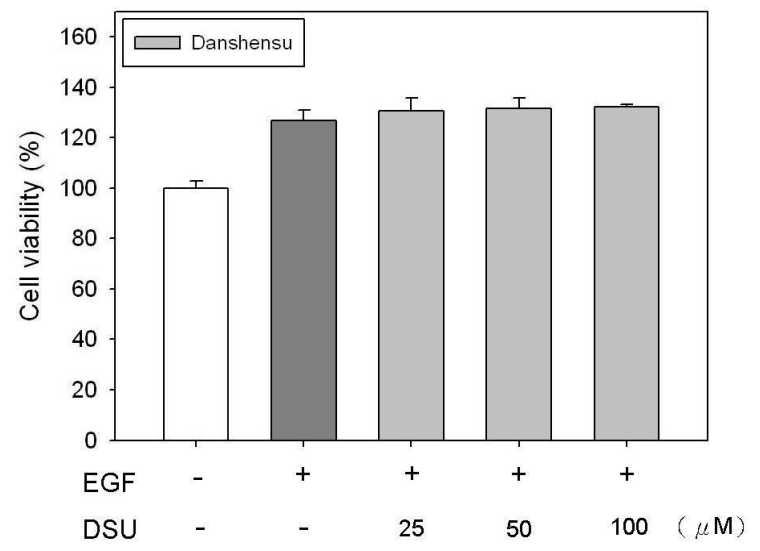

(B)

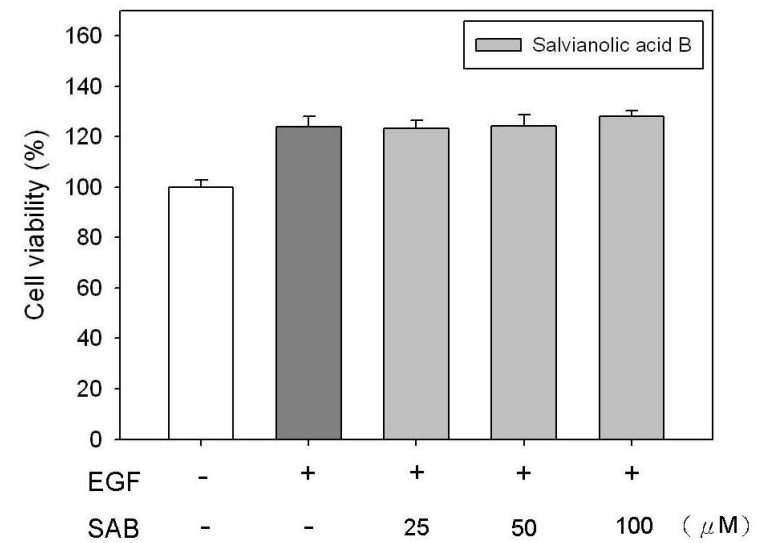

\subsection{Effects of SME, DSU and SAB on the Collagen Production of Fibroblast Cells}

Due to the cell proliferation-enhancing effect of SME, DSU and SAB on fibroblast cells, we hypothesized that the compounds could also enhance the production of collagen in fibroblast cells. Therefore, we analyzed the collagen levels of Detroit 551 cells treated with SME, DSU and SAB, and the results are shown in Figure 4. Our experiments revealed that all of the tested SME, DSU and SAB samples could increase collagen production in Detroit 551 cells. The increased collagen levels in the SME, DSU and SAB treated Detroit 551 cells were all approximately $110 \%$ of the control (Figure 4). Our assay specifically examined the quantities of type I through type $\mathrm{V}$ collagen. Although the increased collagen levels were not quite as high as $110 \%$ after $24 \mathrm{~h}$ treatment, we still believe that SME, DSU and SAB have the ability to improve the production of collagen in fibroblast cells. This is because the effects of SME, DSU and SAB on collagen production were equivalent to those of the positive control (vitamin $\mathrm{C}, 0.3 \mathrm{mM}$ ). Collagen expression is mainly regulated by the transforming growth factor beta (TGF- $\beta$ )/Smads signaling pathway in fibroblast cells. Moreover, the mitogen activated protein kinase (MAPK) signaling pathway is necessary for receptor tyrosine-kinase induced transcription 
of TGF- $\beta$ gene [22]. Therefore, we can propose that the activation of collagen production in SME-, DSU- and SAB-treated Detroit 551 cells were associated with their cell growth-enhancing functions.

Figure 4. The effects of a S. miltiorrhiza extract (SME), danshensu (DSU) and salvianolic acid B (SAB) on the collagen production of Detroit 551 cells. C is the control group and VitC is vitamin $\mathrm{C}$. Each value represents the mean $\pm \mathrm{SE}(n=3) .{ }^{*} p<0.05$, compared with the control.

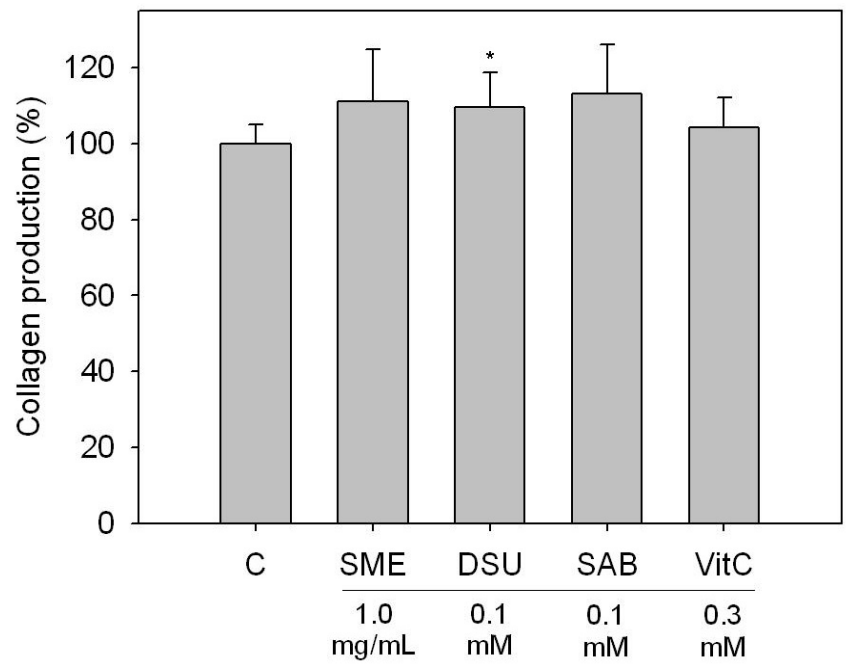

\subsection{Inhibition effects of DSU and SAB on Tyrosinase Activity}

Because the chemical structure of DSU is similar to that of DOPA, we hypothesized that DSU and SAB might have the ability to inhibit tyrosinase activity. The results of this experiment are shown in Figure 5.

Figure 5. The inhibition effects of (A) danshensu and (B) salvianolic acid B on tyrosinase activity. Each value represents the mean $\pm \mathrm{SE}(n=3)$.
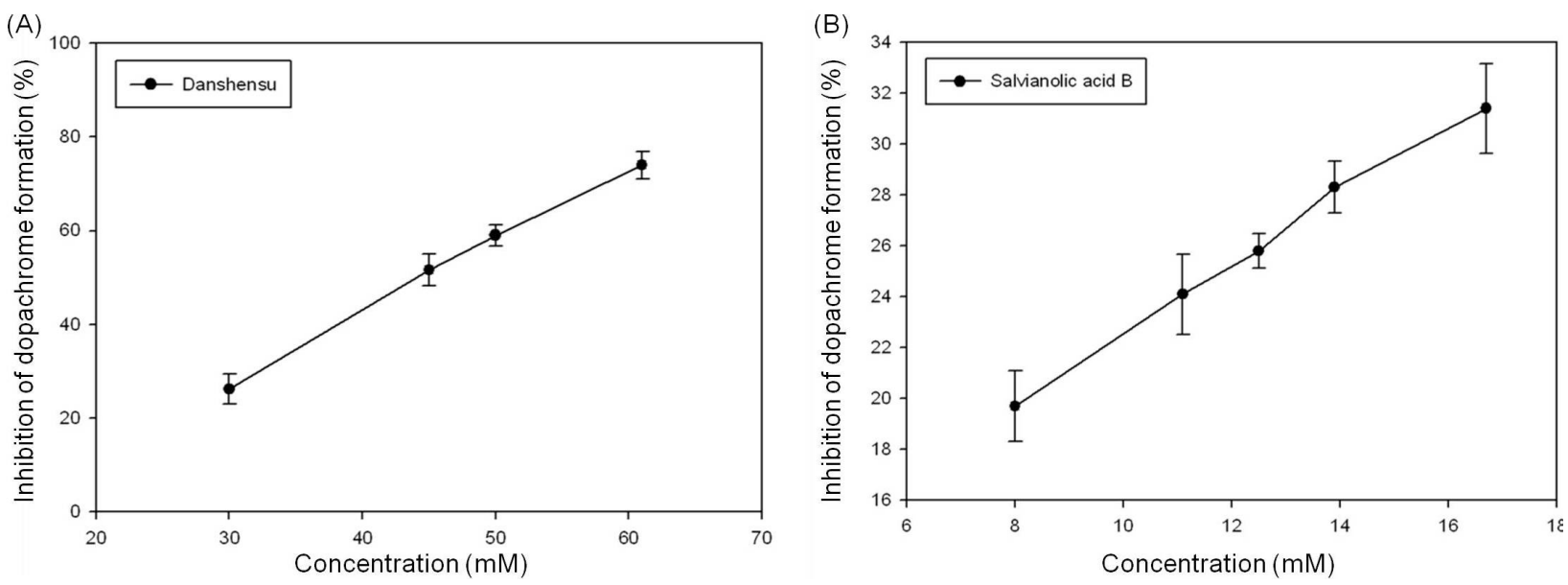

We found that the inhibition of dopachrome formation is increased with increasing DSU concentrations. An inhibition rate of $74 \%$ can be achieved with a DSU concentration of $60 \mathrm{mM}$ (Figure 5A). The calculated $\mathrm{IC}_{50}$ value of DSU on dopachrome formation inhibition is $44.1 \pm 1.1 \mathrm{mM}$. 
In contrast, Figure 5B shows that although $\mathrm{SAB}$ can suppress the inhibition of dopachrome formation in a dose-dependent manner, the suppression ratio only reaches approximately $31 \%$ at a concentration of $17 \mathrm{mM}$ (the highest solubility of SAB in water). Ultimately, our results demonstrated that DSU and $\mathrm{SAB}$ both have the ability to inhibit the activity of tyrosinase, though DSU is more effective than SAB.

\subsection{Inhibition Effects of DSU and SAB on Melanin Production in $\alpha$-MSH-Stimulated Melanoma Cells}

Because the tyrosinase used in our experiment was isolated from mushrooms, the inhibition effects of DSU and SAB on melanin production were further confirmed in a cell based assay model. Therefore, we tested the inhibition effects of DSU and SAB on melanin production in $\alpha-\mathrm{MSH}-$ stimulated B16 melanoma cells. First, we verified the effect of DSU and SAB on the growth of the B16 cells. Those results are shown in Figure 6, and neither DSU nor SAB showed cytotoxicity in B16 cells at the tested concentrations. Moreover, at higher concentrations, DSU and SAB can slightly increase the viability of B16 cells. Although the B16 cell growth-enhancing effects of DSU and SAB are not notable when compared with those of Detroit cells, they were established in our experiments (Figure 6A,B).

Figure 6. The effects of (A) danshensu and (B) salvianolic acid B on the cell viability of B16 melanoma cells. Each value represents the mean $\pm \mathrm{SE}(n=3)$. ${ }^{*} p<0.05$, compared with the control.
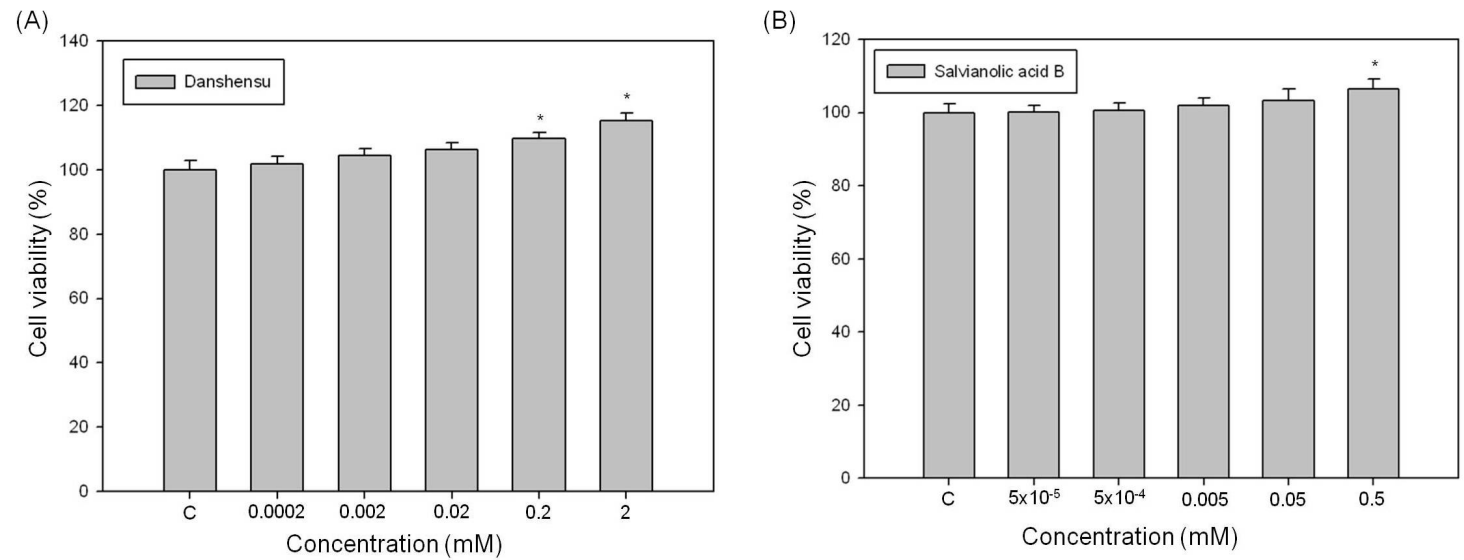

Subsequently, we verified the inhibition effects of DSU and SAB on melanin production in $\alpha$-MSHstimulated B16 melanoma cells, and those results are shown in Figure 7. Under $\alpha$-MSH stimulation, the melanin content was increased to approximately $150 \%$ of the control (untreated B16 cells). Treatment with high concentrations of DSU and SAB decreased the $\alpha$-MSH-stimulated melanin levels. A concentration of $2 \mathrm{mM}$ DSU reduced the $\alpha$-MSH-stimulated melanin content by $15 \%$ (Figure 7A). Treatment with $0.5 \mathrm{mM} \mathrm{SAB}$ attenuated the $\alpha$-MSH-stimulated melanin content by approximately $20 \%$ (Figure 7B). Therefore, both DSU and SAB can suppress melanin production in $\alpha-\mathrm{MSH}-$ stimulated B16 cells. Moreover, the effects of the compounds on melanin production are different from their impact on tyrosinase activity. This difference may have occurred because the tyrosinase used came from mushrooms, and tyrosinases from different species have diverse protein structures that can lead to dissimilar results. 
The synthesis of melanin in melanocytes can be induced by numerous factors, including $\alpha-\mathrm{MSH}$, cyclic adenosine monophosphate (cAMP)-elevating agents (such as isobutylmethylxanthine and forskolin) and UV light [23,24]. Finding agents to regulate melanin synthesis is important for the treatment of hyper- or hypo-pigmentation [25-27]. Our previous study found that SME can strongly enhance tyrosinase activity and melanin production [28]. Thus, although DSU and SAB are the major components of the water extract of S. miltiorrhiza, SME and DSU/SAB can have totally opposite effects on biological functions. For that reason, we suggest that some other functional components in SME strongly increase melanin production in melanoma cells; this effect must be stronger than the inhibition of melanin production provided by DSU and SAB. Regardless, our results continue to demonstrate that DSU and SAB have the potential to treat hyperpigmentation.

Figure 7. The inhibition effects of (A) danshensu (DSU) and (B) salvianolic acid B (SAB) on melanin production in $\alpha$-MSH-stimulated B16 cells. Each value represents the mean \pm $\mathrm{SE}(n=3) .{ }^{*} p<0.05$, compared with the control.
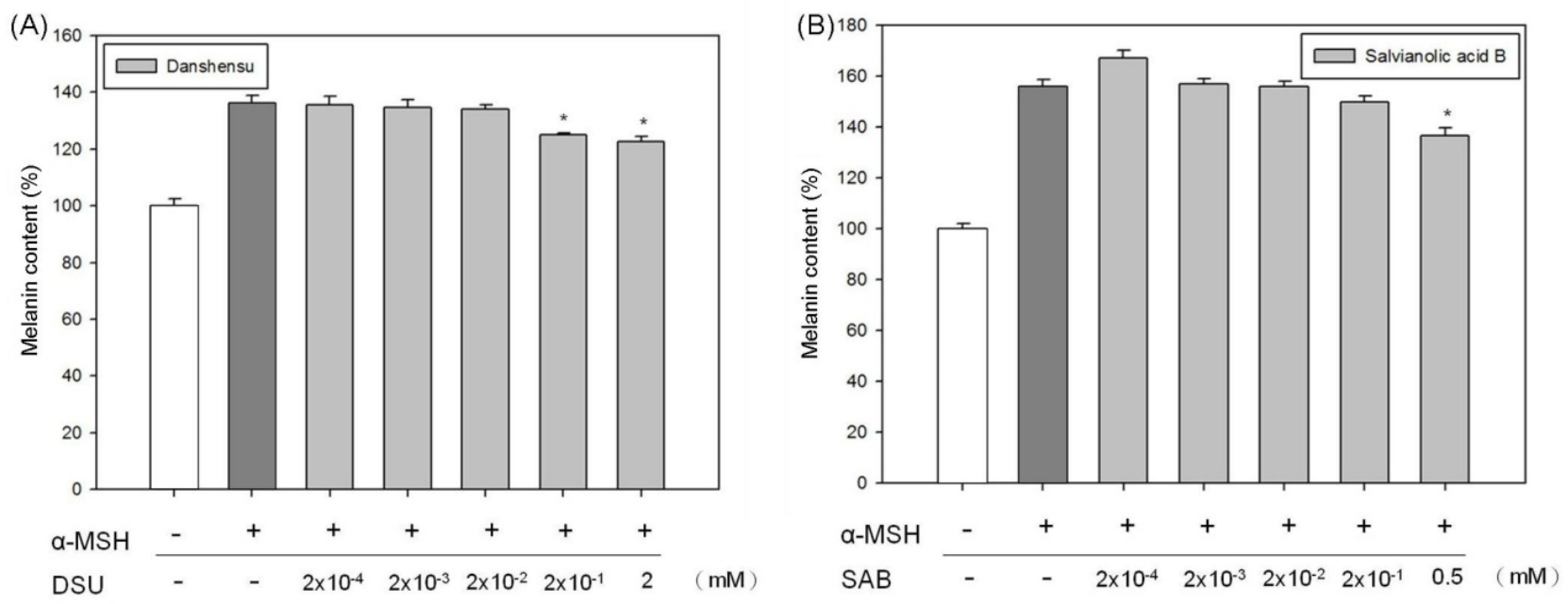

\section{Experimental}

\subsection{Materials}

Salvia miltiorrhiza was provided by WinPower Technology Co., Ltd. (Kaohsiung, Taiwan). Danshensu and salvianolic acid B were purchased from Fusol Material Co., Ltd. (Tainan, Taiwan). Human recombinant epidermal growth factor (EGF) was purchased from ProSpec Inc. (Ness Ziona, Israel). Vitamin C, Triton X-100, mushroom tyrosinase, L-tyrosine, $\alpha$-melanocyte stimulating hormone ( $\alpha$-MSH), dimethyl sulfoxide (DMSO) and other chemicals were purchased from Sigma-Aldrich (St. Louis, MO, USA). Dulbecco's modified Eagle's medium (DMEM), $\alpha$-modified essential medium $(\alpha-M E M)$, fetal bovine serum (FBS), L-glutamine, penicillin-streptomycin and trypsin ethylenediaminetetraacetic acid (trypsin EDTA) were purchased from Invitrogen Life Technologies (Carlsbad, CA, USA). The compound 3-(4,5-dimethylthiazol-2-yl)-2,5-diphenyltetrazolium bromide (MTT) was purchased from Affymetrix/USB (Cleveland, OH, USA). The deionized distilled water $\left(\mathrm{ddH}_{2} \mathrm{O}\right)$ used in solutions and buffers was purified with a Milli-Q system (Millipore, Bedford, MA, USA). 


\subsection{Preparation of Extracts}

The leaves of $S$. miltiorrhiza were cleaned with pure water. After cleaning, the prepared leaves were dried by airing. The dehydrated $S$. miltiorrhiza leaves were homogenized and then extracted with cold water at $25{ }^{\circ} \mathrm{C}$ for $30 \mathrm{~min}$. The collected supernatant was filtered by $0.45 \mu \mathrm{m}$ filters to remove any debris. The filtered S. miltiorrhiza extract (SME) was freeze-dried and stored at $4{ }^{\circ} \mathrm{C}$ prior to use [28].

\subsection{Cell Culture and MTT Assay}

The Detroit 551 normal fibroblast cells (BCRC 60118) and the B16 melanoma cells (BCRC 60031) were purchased from the Food Industry Research and Development Institute (FIRDI, Hsinchu, Taiwan). The Detroit 551 and B16 cells were cultured in $\alpha$-MEM and DMEM, respectively, and then supplemented with $10 \%$ FBS, $2 \mathrm{mM}$ glutamine, $100 \mathrm{mg} / \mathrm{mL}$ streptomycin and $100 \mathrm{U} / \mathrm{mL}$ penicillin. The cells were maintained in a humidified incubator with $5 \% \mathrm{CO}_{2}$ at $37{ }^{\circ} \mathrm{C}$, and they were sub-cultured every 3 to 4 days to maintain logarithmic growth. For the cell viability assays, cells were seeded in a 96-well plate at a density of $5 \times 10^{3}$ cells/well. After $24 \mathrm{~h}$ of incubation, different concentrations of the test compounds or EGF $(0.2 \mu \mathrm{g} / \mathrm{mL})$ were added to each well of the plate, and then the plate was incubated for an additional $24 \mathrm{~h}$. Cell viability was determined via an improved MTT assay [29].

\subsection{Collagen Assay}

The total amount of soluble collagen was assessed using a Sircol Collagen Assay Kit according to the manufacturer's instructions (Biocolor, Carrickfergus, Northern Ireland, UK). In the collagen assay, Detroit 551 cells $\left(6 \times 10^{5}\right.$ cells/well) were incubated in a 6 -well plate with various concentrations of the test compounds for $24 \mathrm{~h}$, and then the cells were collected and lysed using $300 \mu \mathrm{L}$ PBS with $1 \%$ Triton X-100. The samples were centrifuged (12,000 rpm for $10 \mathrm{~min})$, and each $100 \mu \mathrm{L}$ of either the cell sample or a collagen standard was mixed with $1 \mathrm{~mL}$ of Sircol dye for $30 \mathrm{~min}$ and then centrifuged at 10,000 rpm for $5 \mathrm{~min}$ to separate the collagen-dye complex from the supernatant. The collagen-dye complex was dissolved in $1 \mathrm{~mL}$ of Sircol alkali reagent and vortexed. Vitamin C was used as a control. The absorbance of the solution was examined at $540 \mathrm{~nm}$. The quantity of collagen was calculated from the collagen standards and expressed as a percentage of the control [30].

\subsection{Tyrosinase Activity Assay}

For the tyrosinase activity assay, $60 \mu \mathrm{L}$ samples of different concentrations of the test compounds were mixed with $100 \mu \mathrm{L}$ of $1 \mathrm{mM}$ L-tyrosine in phosphate buffer solution (pH 6.8). Then, $40 \mu \mathrm{L}$ of mushroom tyrosinase solution $(100 \mathrm{units} / \mathrm{mL})$ was added to the mixture and incubated for $25 \mathrm{~min}$ at $37^{\circ} \mathrm{C}$. A spectrophotometric analysis was performed at $475 \mathrm{~nm}$, and the inhibition of dopachrome formation was calculated as an inhibition percentage [31]. 


\subsection{Melanin Content Assay}

B16 melanoma cells $\left(2 \times 10^{5}\right.$ cells/well $)$ were incubated in 6 -well plates with various concentrations of the test compounds. The cells were co-treated with $100 \mathrm{nM} \alpha$-MSH for $24 \mathrm{~h}$. After this treatment, the cells were dissolved in $120 \mu \mathrm{L}$ of $1 \mathrm{~N} \mathrm{NaOH}$ for $1 \mathrm{~h}$ at $65^{\circ} \mathrm{C}$ to solubilize the melanin. The total amount of melanin in each cell suspension was determined by recording the absorbance at $405 \mathrm{~nm}$. The melanin content was calculated and corrected for the cell number [32].

\subsection{Statistical Analysis}

All analytical measurements were performed in triplicate. The results were analyzed using Student's $t$-test and were expressed as the mean \pm standard error for each measurement. $p$-values less than 0.05 were considered to be significant.

\section{Conclusions}

In summary, our experiments demonstrated that SME has a growth factor-like ability to enhance the proliferation of Detroit 551 fibroblast cells and that this effect may be caused by DSU and SAB. Additionally, SME, DSU and SAB all have the ability to increase the production of collagen in Detroit 551 cells. Our results also confirmed that DSU and SAB can suppress melanin production in $\alpha$-MSH-stimulated B16 melanoma cells through the inhibition of tyrosinase activity. The proposed mechanisms of DSU and SAB on cell proliferation and collagen and melanin production are shown in Figure 8. Therefore, SME, DSU and SAB have the potential to be used as active ingredients for wound healing and cosmetic treatments. Moreover, DSU and SAB also have the potential to treat hyperpigmentation.

Figure 8. Proposed mechanisms of danshensu (DSU) and salvianolic acid B (SAB) on cell proliferation and collagen and melanin production.

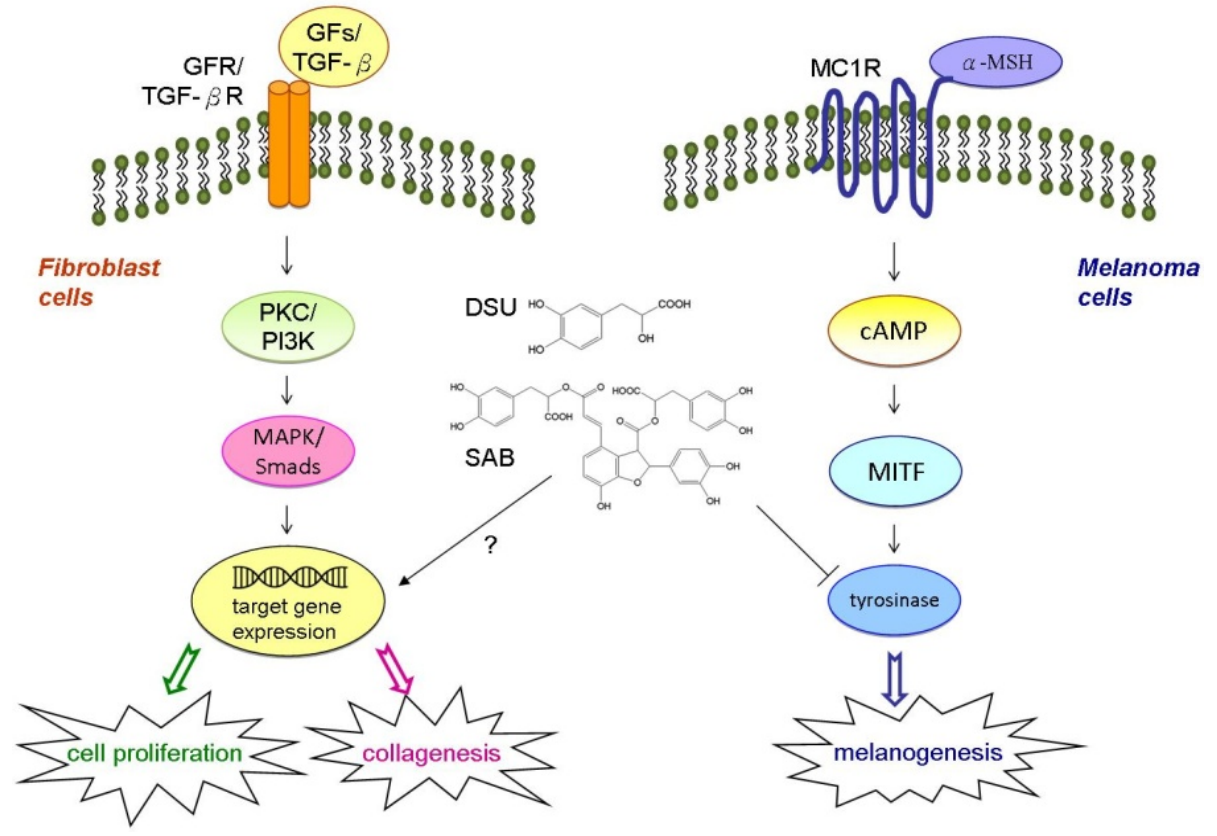




\section{Acknowledgments}

The authors are grateful for financial support from the National Science Council of the Republic of China (Taiwan) awarded to Chih-Chien Lin (NSC102-2313-B-126-001-MY3).

\section{Author Contributions}

Yi-Shyan Chen executed the experiments and prepared the manuscript. Shu-Mei Lee carried out experiments and revised the manuscript. Ying-Ju Lin established the assay system and contributed with valuable discussions. Shu-Hua Chiang and Chih-Chien Lin (corresponding authors) executed the experiments and handled manuscript submission. All authors read and approved the manuscript.

\section{Conflicts of Interest}

The authors declare no conflict of interest.

\section{References}

1. Wu, W.Y.; Wang, Y.P. Pharmacological actions and therapeutic applications of Salvia miltiorrhiza depside salt and its active components. Acta Pharmacol. Sin. 2011, 33, 1119-1130.

2. Ma, P.; Liu, J.; Zhang, C.; Liang, Z. Regulation of water-soluble phenolic acid biosynthesis in Salvia miltiorrhiza Bunge. Appl. Biochem. Biotech. 2013, 170, 1253-1262.

3. Chan, P.; Liu, J.C.; Lin, L.J.; Chen, P.Y.; Cheng, T.H.; Lin, J.G.; Hong, H.J. Tanshinone IIA inhibits angiotensin II-induced cell proliferation in rat cardiac fibroblasts. Am. J. Chin. Med. 2011, 39, 381-394.

4. Cheng, T.O. Cardiovascular effects of Danshen. Int. J. Cardiol. 2007, 121, 9-22.

5. Dong, Y.; Nakagawa-Goto, K.; Lai, C.Y.; Morris-Natschke, S.L.; Bastow, K.F.; Lee, K.H. Antitumor agents 287. Substituted 4-amino-2H-pyran-2-one (APO) analogs reveal a new scaffold from neo-tanshinlactone with in vitro anticancer activity. Bioorg. Med. Chem. Lett. 2011, 21, 2341-2344.

6. Tang, Y.; Wang, M.; Le, X.; Meng, J.; Huang, L.; Yu, P.; Chen, J.; Wu, P. Antioxidant and cardioprotective effects of Danshensu (3-(3, 4-dihydroxyphenyl)-2-hydroxy-propanoic acid from Salvia miltiorrhiza) on isoproterenol-induced myocardial hypertrophy in rats. Phytomedicine 2011, 18, 1024-1030.

7. Zhao, G.R.; Zhang, H.M.; Ye, T.X.; Xiang, Z.J.; Yuan, Y.J.; Guo, Z.X.; Zhao, L.B. Characterization of the radical scavenging and antioxidant activities of danshensu and salvianolic acid B. Food Chem. Toxicol. 2008, 46, 73-81.

8. Hu, P.; Luo, G.A.; Zhao, Z.; Jiang, Z.H. Quality assessment of radix salviae miltiorrhizae. Chem. Pharm. Bull. 2005, 53, 481-486.

9. Cao, Y.; Chai, J.G.; Chen, Y.C.; Zhao, J.; Zhou, J.; Shao, J.P.; Ma, C.; Liu, X.D.; Liu, X.Q. Beneficial effects of danshensu, an active component of Salvia miltiorrhiza, on homocysteine metabolism via the trans-sulphuration pathway in rats. Brit. J. Pharmacol. 2009, 157, 482-490. 
10. Chan, K.; Chui, S.H.; Wong, D.Y.; Ha, W.Y.; Chan, C.L.; Wong, R.N. Protective effects of Danshensu from the aqueous extract of Salvia miltiorrhiza (Danshen) against homocysteineinduced endothelial dysfunction. Life Sci. 2004, 75, 3157-3171.

11. Hao, Y.; Xie, T.; Korotcov, A.; Zhou, Y.; Pang, X.; Shan, L.; Ji, H.; Sridhar, R.; Wang, P.; Califano, J.; et al. Salvianolic acid B inhibits growth of head and neck squamous cell carcinoma in vitro and in vivo via cyclooxygenase-2 and apoptotic pathways. Int. J. Cancer 2009, 124, 2200-2209.

12. Ho, J.H.; Hong, C.Y. Salvianolic acids: Small compounds with multiple mechanisms for cardiovascular protection. J. Biomed. Sci. 2011, 18, 30.

13. Wang, S.X.; Hu, L.M.; Gao, X.M.; Guo, H.; Fan, G.W. Anti-inflammatory activity of salvianolic acid B in microglia contributes to its neuroprotective effect. Neurochem. Res. 2010, 35, 1029-1037.

14. Zhou, Z.T.; Yang, Y.; Ge, J.P. The preventive effect of salvianolic acid B on malignant transformation of DMBA-induced oral premalignant lesion in hamsters. Carcinogenesis 2006, 27, 826-832.

15. Chang, T.-S. Natural melanogenesis inhibitors acting through the down-regulation of tyrosinase activity. Materials 2012, 5, 1661-1685.

16. Li, W.J.; Lin, Y.C.; Wu, P.F.; Wen, Z.H.; Liu, P.L.; Chen, C.Y.; Wang, H.M. Biofunctional constituents from Liriodendron tulipifera with antioxidants and anti-melanogenic properties. Int. J. Mol. Sci. 2013, 14, 1698-1712.

17. Ricard-Blum, S. The collagen family. Cold Spring Harb. Perspect. Biol. 2011, 3, doi:10.1101/cshperspect.a004978.

18. Rossert, J.; Terraz, C.; Dupont, S. Regulation of type I collagen genes expression. Nephrol. Dial. Transplant. 2000, 15 (Suppl.6), 66-68.

19. Han, Y.; Jung, H.W.; Park, Y.K. The roots of Atractylodes japonica Koidzumi promote adipogenic differentiation via activation of the insulin signaling pathway in 3T3-L1 cells. BMC Complement. Altern. Med. 2012, 12, 154.

20. Lin, C.C.; Wu, P.S.; Liang, D.W.; Kwan, C.C.; Chen, Y.S. Quality, antioxidative ability, and cell proliferation-enhancing activity of fermented black soybean broths with various supplemental culture medium. J. Food. Sci. 2011, 77, C95-C101.

21. Morita, T.; Kitagawa, M.; Yamamoto, S.; Suzuki, M.; Sogabe, A.; Imura, T.; Fukuoka, T.; Kitamoto, D. Activation of fibroblast and papilla cells by glycolipid biosurfactants, mannosylerythritol lipids. J. Oleo. Sci. 2010, 59, 451-455.

22. Bhogal, R.K.; Stoica, C.M.; McGaha, T.L.; Bona, C.A. Molecular aspects of regulation of collagen gene expression in fibrosis. J. Clin. Immunol. 2005, 25, 592-603.

23. Koo, J.H.; Rhee, K.S.; Koh, H.W.; Jang, H.Y.; Park, B.H.; Park, J.W. Guggulsterone inhibits melanogenesis in B16 murine melanoma cells by downregulating tyrosinase expression. Int. J. Mol. Med. 2012, 30, 974-978.

24. Slominski, A.; Zmijewski, M.A.; Pawelek, J. L-tyrosine and L-dihydroxyphenylalanine as hormone-like regulators of melanocyte functions. Pigment Cell Melanoma Res. 2012, 25, 14-27. 
25. Chiang, H.M.; Lin, J.W.; Hsiao, P.L.; Tsai, S.Y.; Wen, K.C. Hydrolysates of citrus plants stimulate melanogenesis protecting against UV-induced dermal damage. Phytother. Res. 2011, 25, 569-576.

26. Hamid, M.A.; Sarmidi, M.R.; Park, C.S. Mangosteen leaf extract increases melanogenesis in B16F1 melanoma cells by stimulating tyrosinase activity in vitro and by up-regulating tyrosinase gene expression. Int. J. Mol. Med. 2011, 29, 209-217.

27. Qiao, Z.; Koizumi, Y.; Zhang, M.; Natsui, M.; Flores, M.J.; Gao, L.; Yusa, K.; Koyota, S.; Sugiyama, T. Anti-melanogenesis effect of Glechoma hederacea L. extract on B16 murine melanoma cells. Biosci. Biotechnol. Biochem. 2012, 76, 1877-1883.

28. Chiang, S.-H.; Chen, Y.-S.; Hung, M.-S.; Lee, S.-M.; Lin, C.-C. The enhancement effect of Salvia miltiorrhiza on melanin production of B16F10 melanoma cells. J. Med. Plants Res. 2012, 6, 4338-4342.

29. Chou, S.-T.; Lai, C.-P.; Lin, C.-C.; Shih, Y. Study of the chemical composition, antioxidant activity and anti-inflammatory activity of essential oil from Vetiveria zizanioides. Food Chem. 2012, 134, 262-268.

30. Kim, D.Y.; Kwon, E.Y.; Hong, G.U.; Lee, Y.S.; Lee, S.H.; Ro, J.Y. Cigarette smoke exacerbates mouse allergic asthma through Smad proteins expressed in mast cells. Respir. Res. 2011, 12, 49.

31. Chen, Y.S.; Lee, S.M.; Lin, C.C.; Liu, C.Y.; Wu, M.C.; Shi, W.L. Kinetic study on the tyrosinase and melanin formation inhibitory activities of carthamus yellow isolated from Carthamus tinctorius L. J. Biosci. Bioeng. 2013, 115, 242-245.

32. Huang, H.C.; Chiu, S.H.; Chang, T.M. Inhibitory effect of [6]-gingerol on melanogenesis in B16F10 melanoma cells and a possible mechanism of action. Biosci. Biotechnol. Biochem. 2011, 75, 1067-1072.

Sample Availability: Not available.

(C) 2014 by the authors; licensee MDPI, Basel, Switzerland. This article is an open access article distributed under the terms and conditions of the Creative Commons Attribution license (http://creativecommons.org/licenses/by/3.0/). 